\title{
Substrate Integrated Waveguide with Corrugated Wall
}

Denis G. Chen, and Kimberley W. Eccleston

Department of Electrical and Computer Engineering

University of Canterbury, Christchurch, New Zealand

denischn@gmail.com; kim.eccleston@canterbury.ac.nz

\section{Introduction}

Substrate integrated waveguide (SIW) allows waveguide techniques to be implemented on PCBs [1]. The technology has been successfully applied to a number of devices such as filters, couplers, dividers, slot array antennas [2], oscillators, six port circuits, and circulators [3]. Its models and numerical analysis techniques have also been extensively researched [4].

One particular challenge facing SIW circuits is their integration with active components that require DC biasing. An inherent DC short-circuit is formed between conventional SIWs' top and bottom waveguide plates due to the conducting vias. Several schemes have been proposed to address this issue. They include the use of discrete capacitors on microstrip lines leading up to the SIW circuit [5], embedding an interdigitated capacitor into the SIW-tomicrostrip transition [6], and using AC coupling on half mode SIW [7]. None of the existing methods allow direct shunt connection of active components, such as Gunn diodes, into a waveguide. Compromises are usually made to accommodate the need of DC bias; and consequently, the advantages of SIW structure are not fully utilized and the solution is suboptimal.

In this work we propose a new SIW structure, called the corrugated SIW (CSIW), which does not require conducting vias to achieve $\mathrm{TE}_{10}$ type boundary conditions at the side walls. Instead, the vias are replaced by quarter wavelength microstrip stubs arranged in a corrugated pattern on the edges of the waveguide. This, along with series interdigitated capacitors [6], results in a waveguide section comprising two separate conductors, which facilitates shunt connection of active components such as Gunn diodes [8]. The concept of this arrangement is shown in Fig. 1.

\section{Proposed Corrugated Substrate Integrated Waveguide}

The layout of a CSIW and its critical dimensions are given in Fig. 2. The waveguide width $(a)$, and $50 \Omega$ microstrip to SIW transition are designed according to previously described methods $[3,9]$. They can be fine tuned and optimized separately by way of EM simulations.

The length of the corrugated wall stubs $(t)$ is approximately one quarter of the TEM guided wavelength $\left(\lambda_{\mathrm{g}}\right)$ at the centre frequency. This estimation is refined by EM simulation which accounts for fringing effects and transition from $\mathrm{TE}_{10}$ mode to quasi-TEM mode at the side wall.

Different combinations of stub spacing $(u)$ and width $(v)$ have been investigated. We conclude that they have very little impact on the overall performance of the CSIW. Rules for choosing equivalent dimensions in conventional SIWs can be applied here and values suitable for fabrication should be adopted for the final design. 


\section{Application of CSIW in X-band}

Fig. 3 shows a set of back-to-back SIWs, corrugated and conventional, fabricated on 60 mil thick Taconic RF-30 $\left(\varepsilon_{r}=3.0\right)$ PCB with $35 \mu \mathrm{m}$ copper layer on both sides. Two sets of SIWs were made with different waveguide section lengths: one $81 \mathrm{~mm}$ and another $46.5 \mathrm{~mm}$.

The SIWs were designed for X-band (8.2 GHz to $12.4 \mathrm{GHz}$ ), and the resulting structure parameters are given in Table 1. The internal width $(a)$ and the microstrip transitions are the same for both SIW and CSIW. The parameters $d$ and $s$ refer to the via diameter and spacing for the conventional SIW respectively. The substrate thickness, and hence waveguide height, is denoted as $b$. The surface current density plot for both SIW and CSIW at $10 \mathrm{GHz}$ is shown in Fig. 4 and this is consistent with the $\mathrm{TE}_{10}$ mode.

\begin{tabular}{|c|c|c|c|c|c|c|c|c|c|c|c|}
\hline $\mathrm{a}$ & $\mathrm{b}$ & $\varepsilon_{\mathrm{r}}$ & $\mathrm{d}$ & $\mathrm{s}$ & $\mathrm{m}_{1}$ & $\mathrm{~m}_{\mathrm{w}}$ & $\mathrm{W}$ & $\mathrm{l}$ & $\mathrm{u}$ & $\mathrm{v}$ & $\mathrm{t}$ \\
\hline 13.5 & 1.524 & 3.0 & 1.0 & 2.0 & 4.0 & 4.0 & 7.0 & 17.5 & 1.0 & 1.0 & 5.5 \\
\hline
\end{tabular}

S-parameter measurements were performed using an Agilent E8362B $20 \mathrm{GHz}$ vector network analyzer. Results for the $46.5 \mathrm{~mm}$ waveguides are given in Fig. 5. Measured return loss is better than $10.3 \mathrm{~dB}$ for CSIW and $8.6 \mathrm{~dB}$ for SIW; global insertion loss is lower than $3.5 \mathrm{~dB}$ for CSIW and $2.9 \mathrm{~dB}$ for SIW.

Based on the multiline calibration method [10], the propagation constants (Fig. 6) were extracted from the measurements of the two lengths of CSIW and SIW. The attenuation constant is less than $0.48 \mathrm{~dB} / \mathrm{cm}$ for CSIW and $0.45 \mathrm{~dB} / \mathrm{cm}$ for SIW.

The discrepancies between measurement and simulation are largely attributed by the irregularities in the fabrication process; nonetheless, the results are sufficient to confirm that the CSIW offers performance comparable to that of the conventional SIW.

The interdigitated capacitor on the DC decoupled CSIW (Fig. 7) was designed following a method similar to [6]. It uses 13 fingers of $3.2 \mathrm{~mm}$ length, and a gap of $0.3 \mathrm{~mm}$. Measured results (Fig. 8) suggest that the two capacitors increased insertion loss by approximately 0.5 dB overall when compared to CSIW results in Fig. 5.

\section{Conclusion}

A novel corrugated SIW has been shown to have comparable performance to the conventional SIW. It has the additional advantage of complete DC isolation between its top and bottom plates. This permits shunt connection of active components in the waveguide for a variety of applications.

\section{Acknowledgement}

The authors would like to thank Nick Smith, David Healy, and Michael Cusdin for their assistance in making the PCBs and test fixtures. 


\section{References}

[1] H. Uchimura, T. Takenoshita, and M. Fujii, "Development of a "laminated waveguide", IEEE Trans. Microw. Theory Tech., Vol.46, No.12, pp.2438-2443, Dec. 1998.

[2] W. Hong, "Development of microwave antennas, components and subsystems based on SIW technology," IEEE Int. Symp. on Microw., Antenna, Propagat. and EMC Technologies for Wireless Commun., 2005. Vol.1, pp. P-14-17, 8-12 Aug. 2005

[3] M. Bozzi, F. Xu, D. Deslandes, and K. Wu, "Modeling and Design Considerations for Substrate Integrated Waveguide Circuits and Components," in 8th Int. Telecommun. Modern Satellite, Cable, Broadcast. Service. Conf., 2007, pp.P-VII-P-XVI, 26-28 Sept. 2007.

[4] F. Xu, Y. Zhang, W. Hong; K. Wu, T. J. Cui, "Finite-difference frequency-domain algorithm for modeling guided-wave properties of substrate integrated waveguide," IEEE Trans. Microw. Theory Tech., Vol.51, No.11, pp. 2221-2227, Nov. 2003.

[5] Y. Cassivi and K. Wu, "Low Cost Microwave Oscillator Using Substrate Integrated Waveguide Cavity," IEEE Microw. Wireless Compon. Lett., Vol. 13, No. 2, pp. 48-50, Feb. 2003.

[6] M. Abdolhamidi, A. Enayati, M. Shahabadi, and R. Faraji-Dana, "Wideband singlelayer DC-decoupled substrate integrated (SIW) - to - microstrip transition using an interdigital configuration," 2007 Asia-Pacific Microwave. Conference, pp. 1599-1602.

[7] C. Zhong, J. Xu, Z. Yu, M. Wang, Y. Zhu, "Half Mode Substrate Integrated Waveguide Gunn Oscillator," Global Symp. on Millimeter Waves Proc. 2008, pp.14-16, 21-24 Apr. 2008.

[8] K. Chang, I. Bahl, and V. Nair, RF and Microwave Circuit and Component Design for Wireless Systems. Hoboken, NJ : Wiley, 2002, pp.370-375.

[9] J.-X. Chen, W. Hong, Z.-C. Hao, H. Li, and K. Wu, "Development of a Low Cost Microwave Mixer Using a Broadband Substrate Integrated Waveguide (SIW) Coupler," IEEE Microw. Wireless Compon. Lett., Vol. 16, No. 2, pp.84-86, Feb. 2006.

[10] R. B. Marks, "A multiline method of network analyzer calibration," IEEE Trans. Microw. Theory Tech., Vol.39, No.7, pp.1205-1215, Jul. 1991.

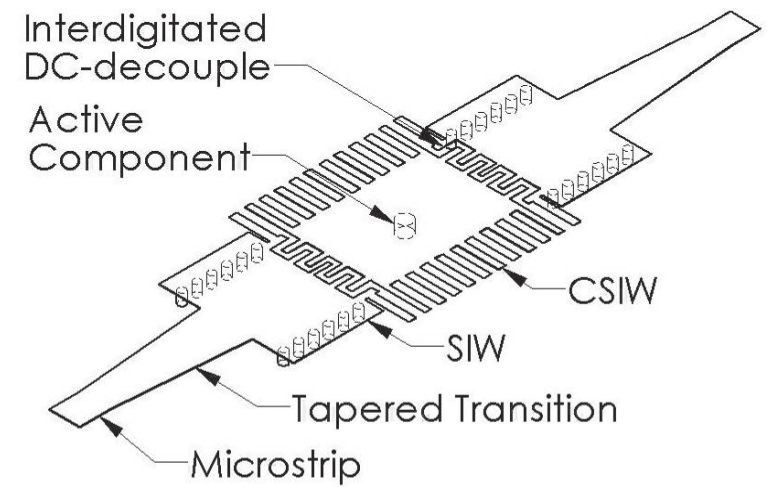

Figure 1. DC decoupled CSIW with embedded component.

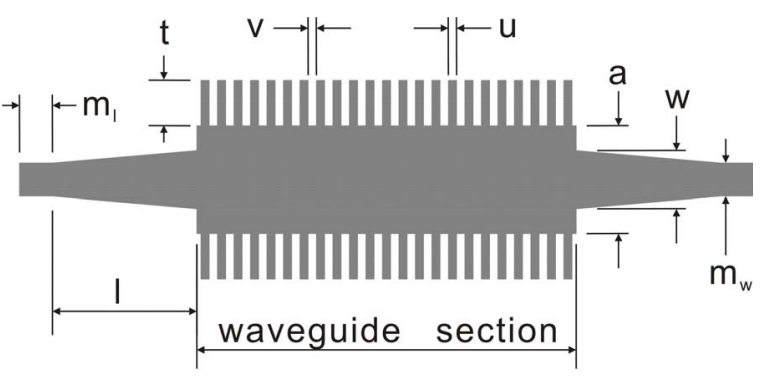

Figure 2. CSIW layout. 


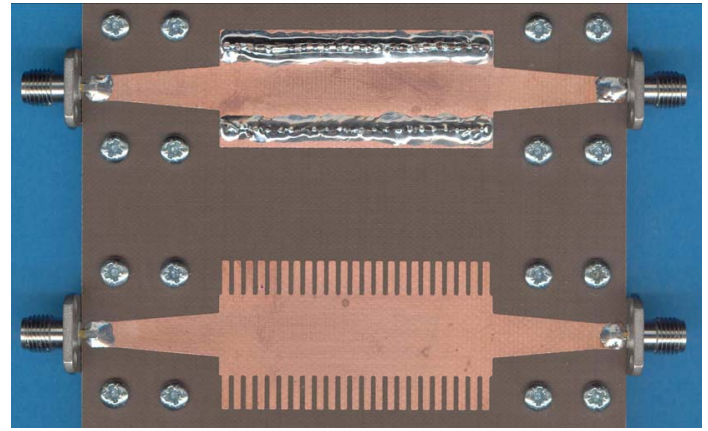

Figure 3. SIW and CSIW with $46.5 \mathrm{~mm}$ waveguide length.

(a)

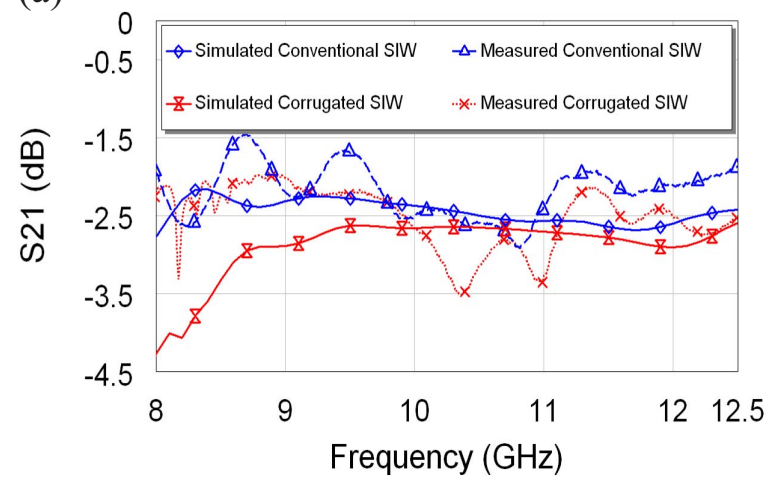

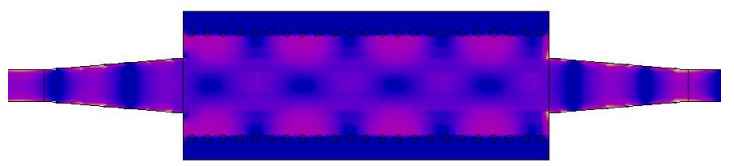

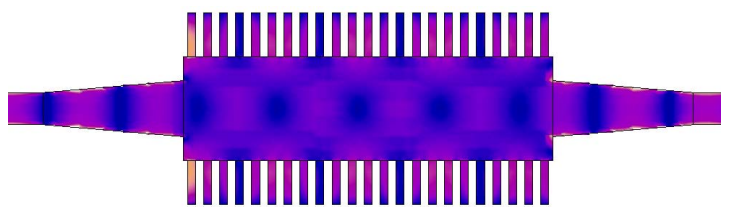

Figure 4. Surface current density plot of SIW and CSIW at $10 \mathrm{GHz}$.

(b)

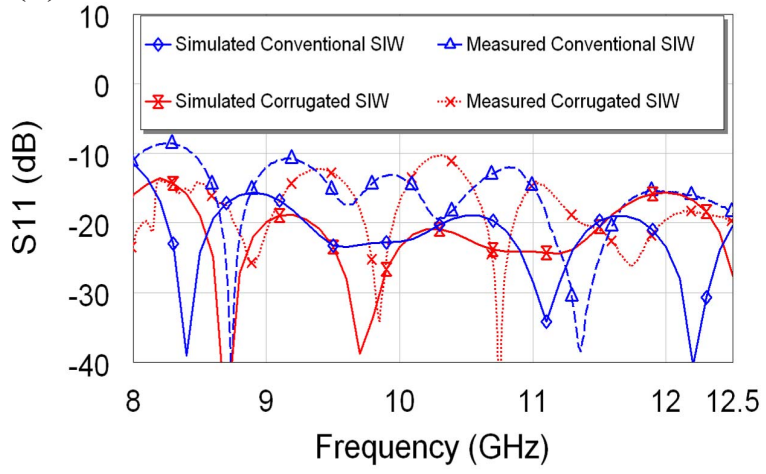

Figure 5. S-parameters of the waveguides. (a) S21. (b) S11.

(a)

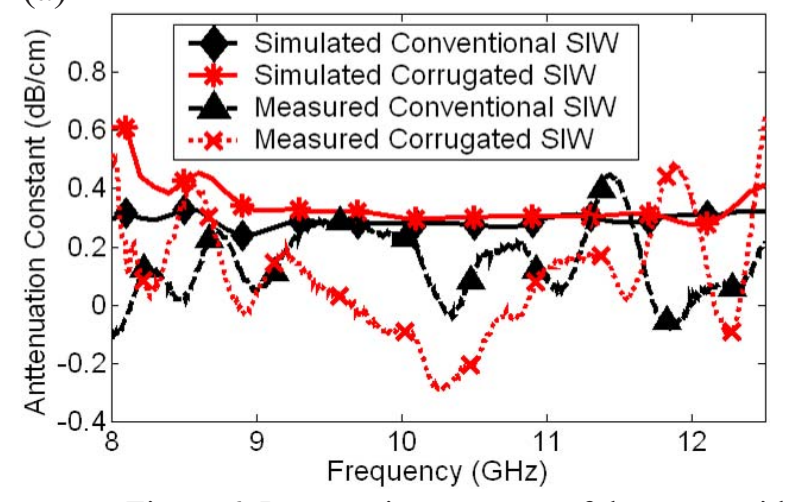

(b)

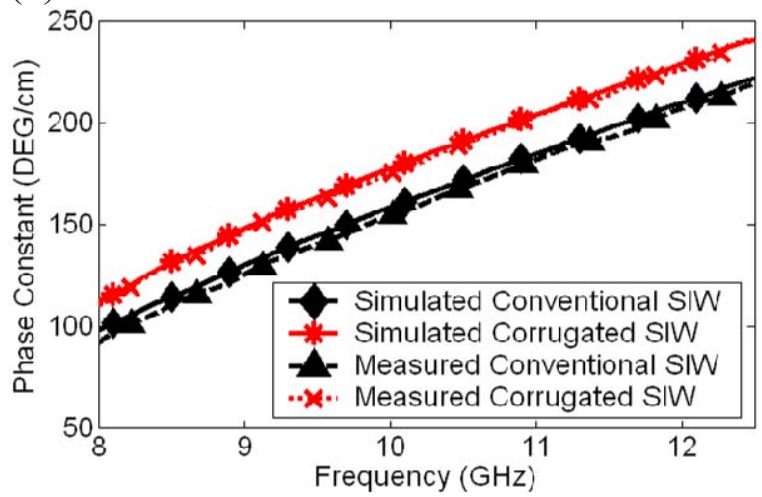

Figure 6. Propagation constant of the waveguides. (a) Attenuation constant. (b) Phase constant.

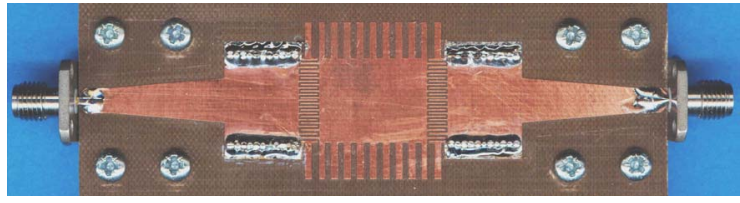

Figure 7. DC decoupled CSIW with $46.5 \mathrm{~mm}$ total waveguide length.

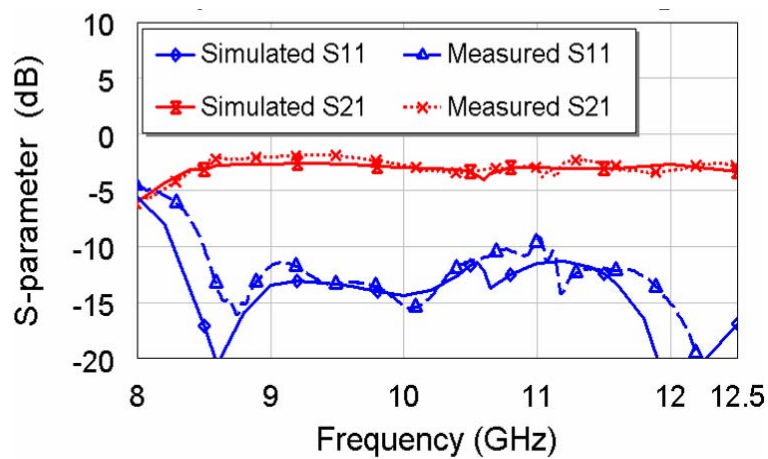

Figure 8. S-parameters of the DC decoupled CSIW. 